\title{
Linfluence de quelques facteurs sur la résistance résiduelle des argiles
}

\section{A. ROUAIGUIA}

Institut de génie civil.

Centre universitaire

de Guelma BP 401

Guelma (24000). Algeria
On étudie ici les effets de la contrainte normale, de la teneur en argile, de la vitesse de cisaillement et du taux d'humidité sur la résistance résiduelle de diverses argiles en utilisant comme dispositif une boîte de cisaillement modifiée.

Tous les essais ont été effectués dans des conditions de consolidation normale. Les échantillons avaient pour dimensions $10 \times 10 \mathrm{~cm}^{2}$ et $2 \mathrm{~cm}$ d'épaisseur.

Les résultats expérimentaux montrent que la composition en argile et l'indice de plasticité ont un effet plus significatif sur l'angle de frottement résiduel que les autres facteurs.

\section{The influence of some factors on the residual shear strength of clays}

This investigation was an examination of the effects of norma stress, clay fraction, rate of shear and moisture content on the residual strength of various clays using reversal modified shearbox apparatus. All the experiments were carried out under normally consolidated conditions, the specimen size is $10 \times 10 \mathrm{~cm}^{2}$, and $2 \mathrm{~cm}$ thick.

Test results indicate that the clay fraction and plasticity index seem to have a significant effect on the residual friction angle more than the other factors.
} 


\section{Introduction}

La résistance résiduelle drainée des sols cohérents est une propriété importante en géotechnique. Son rôle est de définir la perte de résistance notamment dans les argiles fissurées, Elle représente également la résistance disponible le long des surfaces de cisaillement qui se forment dans les sols cohérents. Lorsque le sol a été sujet à des mouvements importants ayant conduit à la formation de plans de glissement, la résistance résiduelle devient une donnée nécessaire à toute étude de projet de construction.

La résistance résiduelle joue un grand rôle dans le comportement des terrairns en éboulement et sa connaissance est très importante dans la conception des murs de soutènement. Elle représente également un facteur important pour évaluer le risque dû à la rupture progressive dans les problèmes de stabilité en général.

La nécessitè de comprendre les mécanismes de base dans le comportement des terrains en éboulement ainsi que d'autres problèmes de géotechnique de l'ingénieur sont à l'origine du développement des connaissances sur la résistance résiduelle. Plusieurs facteurs ont été proposés pour évaluer la résistance résiduelle.

Voight (1973) a fait référence à la corrélation entre les limites d'Atterberg en plasticité et la résistance résiduelle au cisaillement des sols naturels et a établi une relation entre l'indice de plasticité et le coefficient de résistance résiduelle $\mu_{r}^{\prime}$.

Contrairement, Kenney (1967) a indiqué qu'aucune relation n'existe entre la résistance résiduelle et la plasticité du sol. Skempton (1964) a décrit un cas concernant « l'argile de Londres ») dont la teneur en eau était de $34 \%$ au voisinage de la surface de glissement et de $30 \%$ loin de la surface cisaillée. Il a également indiqué que durant le processus de cisaillement, il se formait une bande continue à l'intérieur de laquelle les particules d'arqile étaient fortement orientées dans la direction du cisaillement et formaient un domaine composé de surfaces de glissement parmi lesquelles on distingue nettement la surface de glissement principale.

Blondeau et Josseaume (1976) ont présenté une relation similaire entre la résistance résiduelle et le pourcentage d'argile. Petley (1966) a présenté une étude dans laquelle, pour la première fois, les effets de la vitesse sur la résistance résiduelle étaient systématiquement analysés dans des conditions de déformation contrôlée. Herrmann et Wolfskill (1966) ont indiqué que la vitesse de cisaillement a un effet faible mais perceptible sur les déformations nécessaires pour atteindre les conditions résiduelles pour des schistes à faible pourcentage d'argile. Des vitesses de cisaillement de l'ordre de ( $5 \mathrm{~mm} / \mathrm{mn}$ ) étaient considérées comme trop rapides pour que les conditions résiduelles soient correctement établies.

Dans beaucoup de cas, la contrainte normale moyenne enregistrée sur les plans de rupture est peu élevée et d'après James (1970), atteint rarement les $70 \mathrm{kPa}$. Hawkins et Privet (1985) ont confirmé, d'après leurs expérimentations, que les enveloppes de rupture résiduelle sont courbes et que la courbure est plus prononcée au-dessous d'une contrainte effective d'environ $200 \mathrm{kPa}$ et dans des sols dont le pourcentage d'argile est élevé.

L'objet de cet article est d'étudier l'influence de différents facteurs sur l'angle de frottement résiduel.

\section{2.}

\section{Description de la boîte de cisaillement modifiée}

L'utilisation des systèmes asservis par ordinateur dans les laboratoires de mécanique des sols est maintenant bien établie. La boîte de cisaillement standard a été modifiée comme suit :

a) Le système d'engrenages a été remplacé par un moteur dont le contróle se fait directement à partir d'un micro-ordinateur, en fixant le nombre de pulsations nécessaires pour mettre en marche le moteur. Une vitesse supérieure allant jusqu'à $53 \mathrm{~mm} / \mathrm{mn}$ peut ètre obtenue avec ce dispositif.

b) L'anneau de chargement et les comparateurs sont remplacés par des capteurs électroniques qui mesurent les déplacements horizontal et vertical de la boîte automatiquement à partir du micro-ordinateur.

c) La contrainte normale peut être appliquée par une pression d'air produite par une cellule de chargement contrôlée par le micro-ordinateur (Rogers et al. 1989). Le système d'asservissement est utilisé pour enregistrer les mesures obtenues à l'aide de la boite de cisaillement. Les modifications permettent également d'inverser automatiquement la direction du déplacement à une distance choisie (Rouaiguia, 1990).

\section{3}

\section{Programme expérimental}

\section{1}

\section{Description des échantillons de sol}

Six échantillons d'argile ont été utilisés dont trois provenaient de l'Est algérien et trois autres, composés

tálEaU Propriétés des argiles. Clay properties.

\begin{tabular}{|c|c|c|c|c|c|c|}
\hline & $\begin{array}{l}\text { Aruile de } \\
\text { Landres }\end{array}$ & Aruille diu & $\begin{array}{l}\text { Mame du } \\
\text { Keuper }\end{array}$ & Kanlint & Kaolinz & Kaotin3 \\
\hline $\begin{array}{l}\text { Densité de } \\
\text { grains solides }\end{array}$ & 2,75 & 2,5 & 2.8 & 3.2 & 2.69 & 2.6 \\
\hline $\begin{array}{l}\text { Limite de } \\
\text { liquidité }\end{array}$ & $87 \%$ & $45 \%$ & $35 \%$ & $104 \%$ & $86 \%$ & $57 \%$ \\
\hline $\begin{array}{l}\text { Limite de } \\
\text { plasticité }\end{array}$ & $31 \%$ & $27 \%$ & $17 \%$ & $48 \%$ & $49 \%$ & $36 \%$ \\
\hline $\begin{array}{l}\text { Indice de } \\
\text { plasticité }\end{array}$ & $56 \%$ & $18 \%$ & $18 \%$ & $56 \%$ & $37 \%$ & $21 \%$ \\
\hline Activité & 1,43 & 0,54 & 0,66 & 0,98 & 1.6 & 1,61 \\
\hline $\begin{array}{l}\text { Sable } \\
>0,063 \mathrm{~mm}\end{array}$ & $4 \%$ & $1 \%$ & $12 \%$ & $2 \%$ & $35 \%$ & $42 \%$ \\
\hline $\begin{array}{l}\text { Sables gros } \\
0,063-0,020 \mathrm{~mm}\end{array}$ & $15 \%$ & $26 \%$ & $26 \%$ & $9 \%$ & $2 \%$ & $26 \%$ \\
\hline $\begin{array}{l}\text { Limons moyens } \\
0,02-0,006 \mathrm{~mm}\end{array}$ & $4 \%$ & $8 \%$ & $12 \%$ & $11 \%$ & $28 \%$ & $10 \%$ \\
\hline $\begin{array}{l}\text { Limons fins } \\
0,006-0,002 \mathrm{~mm}\end{array}$ & $38 \%$ & $32 \%$ & $23 \%$ & $21 \%$ & $12 \%$ & $9 \%$ \\
\hline $\begin{array}{l}\text { Argiles } \\
<0,002 \mathrm{~mm}\end{array}$ & $39 \%$ & $33 \%$ & $27 \%$ & $57 \%$ & $23 \%$ & $13 \%$ \\
\hline
\end{tabular}


tABLEAUII Composition minéralogique utilisant la diffraction des rayons X.

Clay mineralogy compositions using $\mathrm{X}$-ray diffraction.

\begin{tabular}{|c|c|}
\hline Echantillon & $\begin{array}{l}\text { Composition minèralocioue } \\
\text { par ordre de domination }\end{array}$ \\
\hline Argile de Londres & Smectite Illite/Mica Chlorite \\
\hline Argile du Lias & Illite/Mica Kaolinite Chlorite \\
\hline Marne du Keuper & Chlorite Illite/Mica \\
\hline Kaolin1 & $\begin{array}{l}\text { Kaolinite dans des couches ordonnées } \\
\text { Illite/Mica very little }\end{array}$ \\
\hline Kaolin2 & $\begin{array}{l}\text { Kaolinite dans des couches bien ordonnées } \\
\text { Illite/Mica very little }\end{array}$ \\
\hline Kaolin3 & $\begin{array}{l}\text { Kaolinite dans des couches moins ordonnées } \\
\text { thlite/Mica płus en comparaison avec le Kaolin1 et } 2\end{array}$ \\
\hline
\end{tabular}

d'argile britannique, avaient été prélevés en Angleterre. Les résultats de l'identification et les compositions minéralogiques des argiles obtenus par diffraction des rayons X sont reportès dans les tableaux I et II.

\section{4}

\section{Choix des vitesses dans les expériences de cisaillement}

La vitesse de cisaillement joue un grand rôle dans l'explication du mouvement des talus et des mécanismes mis en jeu dans tous les problèmes de rupture ou d'instabilité. L'influence de la vitesse de cisaillement sur " l'argile de Londres $»$ et "L'argile du Lias » a été décrite.

Les effets de la vitesse étaient analysés en augmentant la vitesse de cisaillement au cours de chaque cycle de retour, avec une vitesse constante suffisamment faible pour chaque cycle d'aller jusqu'à l'établissement des conditions résiduelles.

L'argile était préparée dans un état d'humidité proche de la limite de liquidité. L'échantillon était d'abord consolidé à une contrainte normale de $50 \mathrm{kPa}$ avant l'application de deux étapes de consolidation pour atteindre successivement les contraintes normales de $100 \mathrm{kPa}$ et $200 \mathrm{kPa}$. L'intervalle de temps entre l'application de ces contraintes normales était d'environ 30 minutes. Afin d'éviter toute perte de ce matériau relativement liquide, sous l'effet de la compression, à travers l'ouverture située entre les deux parties de la boîte, il a été décidé de ne pas appliquer directement la contrainte normale de $200 \mathrm{kPa}$. En plus, les particules d'argiles pouvaient se réarranger et l'eau contenue dans l'échantillon pouvait s'infiltrer normalement.

Une fois l'étape de consolidation terminée, un déplacement de cisaillement de $10 \mathrm{~mm}$ était imposé à une vitesse de $0,00881 \mathrm{~mm} / \mathrm{mn}$ pour le premier cycle de retour. Au cours du premier cycle d'aller, la boîte se déplaçait avec la même vitesse de $0,00881 \mathrm{~mm} / \mathrm{mn}$ : les différentes étapes de l'essai sont indiquées dans le tableau III.
TABLAuIII Vitesse de cisaillement pour chaque cycle. Rate of shear for each cycle.

\begin{tabular}{l|c|c}
\hline Premier cycle & $\begin{array}{c}\text { Vitesse de cisaillement } \\
\text { daller (mm/mm) }\end{array}$ & $\begin{array}{c}\text { Vitesse de cisaillement } \\
\text { de retour (min/mn) }\end{array}$ \\
\hline Deuxième cycle & 0,00881 & 0,00881 \\
\hline Troisième cycle & 0,00881 & 0,01321 \\
\hline Quatrième cycle & 0,00881 & 0,019822 \\
\hline Cinquième cycle & 0,00881 & 0,029733 \\
\hline
\end{tabular}

\section{1}

\section{Influence de la contrainte normale}

La boite de cisaillement a été sujette à un certain nombre de critiques, dans la littérature, à cause de la perturbation de la zone de cisaillement au cours du mouvement inverse de la boitte. Dans le cas de « l'argile de Londres $)$ et de " l'argile du Lias $»$ une tentative a été faite pour étudier l'effet de la perturbation de l'échantillon sur la résistance résiduelle au cisaillement. L'échantillon a été consolidé à l'intérieur de la boîte, sous une contrainte normale effective de $200 \mathrm{kPa}$, le temps alloué pour la consolidation était d'environ 48 heures.

Durant le premier cycle d'aller, une faible vitesse de cisaillement de $0,00881 \mathrm{~mm} / \mathrm{mn}$ était appliquée, suivie d'une réduction de la charge normale jusqu'à zéro sans causer aucune perturbation à l'échantillon. La partie supérieure de la boîte de cisaillement était rapidement ramenée à sa position initiale, à la vitesse de cisaillement de $0,03968 \mathrm{~mm} / \mathrm{mn}$. La contrainte normale était alors appliquée à nouveau et il fallait environ 12 heures de temps pour permettre la consolidation de l'échantillon. Ces opérations étaient répétées jusqu'à un déplacement cumulé suffisant pour atteindre les conditions résiduelles. La variation de la vitesse de cisaillement et de la contrainte normale pour chaque cycle est présentée dans le tableau IV.

TABIEAUIV Variation de la vitesse de cisaillement et de la contrainte normale pour chaque cycle.

The variation of rate of shear and normal stress for each cycle.

\begin{tabular}{l|c|c|c|c}
\hline & $\begin{array}{c}\text { Vitesse de } \\
\text { eisaillement } \\
\text { d'aller } \\
\text { (mmimn) }\end{array}$ & $\begin{array}{c}\text { Vitesse de } \\
\text { cisallement } \\
\text { de retour } \\
\text { (mm/mn) }\end{array}$ & $\begin{array}{c}\text { Contrainte } \\
\text { normale } \\
\text { d'aller } \\
\text { (kPa) } \\
\text { Premier cycle }\end{array}$ & $\begin{array}{c}\text { Contrainte } \\
\text { nomale } \\
\text { de retour } \\
(\mathrm{kPa})\end{array}$ \\
\hline Deuxième cycle & 0,00881 & 0,03968 & 200 & 0 \\
\hline Troisième cycle & 0,00881 & 0,03968 & 200 & 0 \\
\hline Quatrième cycle & 0,00881 & 0,03968 & 200 & 0 \\
\hline Cinquième cycle & 0,00881 & 0,03968 & 200 & 0
\end{tabular}

\section{2}

\section{Mesures du taux d'humidité}

A la fin de chaque essai à l'aide de ce dispositif modifié et réversible, de la boîte de cisaillement, cinq mesures de teneur en eau étaient effectuées sur chaque échantillon. Ces mesures étaient faites sur des prélèvements d'environ $1 \mathrm{~mm}$ d'épaisseur, contenant le plan de cisaillement. 
TADEEAUY Taux d'humidité, angle de frottement et teneur en argile pour chaque échantillon. Final moisture content, residual friction angle and clay fraction for each clay.

\begin{tabular}{|c|c|c|c|}
\hline Araile & $\begin{array}{l}\text { Taux dhumidite } \\
\text { fnal } \\
\text { (\%) }\end{array}$ & $\begin{array}{c}\text { Anole de frottement } \\
\text { résiduel } \\
\text { o' (degrés) }\end{array}$ & $\begin{array}{l}\text { Teneur } \\
\text { en argile } \\
(\%)\end{array}$ \\
\hline Kaolin 1 & 43 & 11,8 & 57 \\
\hline Kaolin 2 & 45 & 17,6 & 23 \\
\hline Kaolin 3 & 34 & 22,8 & 13 \\
\hline Argile de Londres & 32 & 8,2 & 39 \\
\hline Argile du Lias & 33,5 & 12,1 & 33 \\
\hline Marne du Keuper & 22,8 & 22,4 & 27 \\
\hline
\end{tabular}

Le tableau $V$ donne la valeur moyenne des cing mesures finales du taux d'humidité pour chaque argile ainsi que les valeurs de l'angle de frottement résiduel et la composition en argile.

\section{5}

\section{Discussion des résultats}

\author{
5.1
}

\section{Effets de la vitesse de cisaillement et de la contrainte normale}

Les effets de la contrainte normale et de la vitesse de cisaillement étaient étudiés pour " l'argile de Londres » ainsi que pour " l'argile du Lias ». Ces expérimentations étaient effectuées afin d'essaver de minimiser l'effet des perturbations sur la résistance résiduelle.

A partir des résultats obtenus, la diminution de la résistance résiduelle pour " l'argile de Londres » et "l'argile du Lias » a été de 0,3 et 0,27 degrés respectivement.

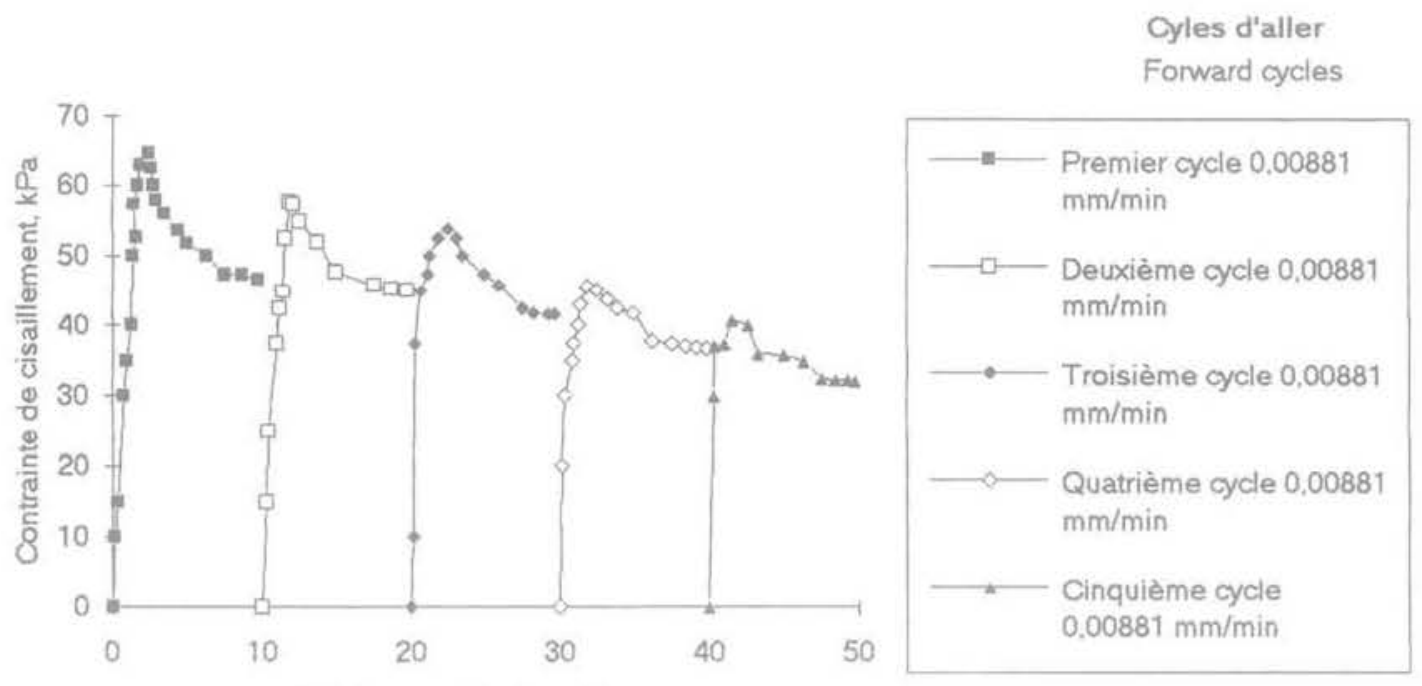

Déplacement horizontal, $\mathrm{mm}$

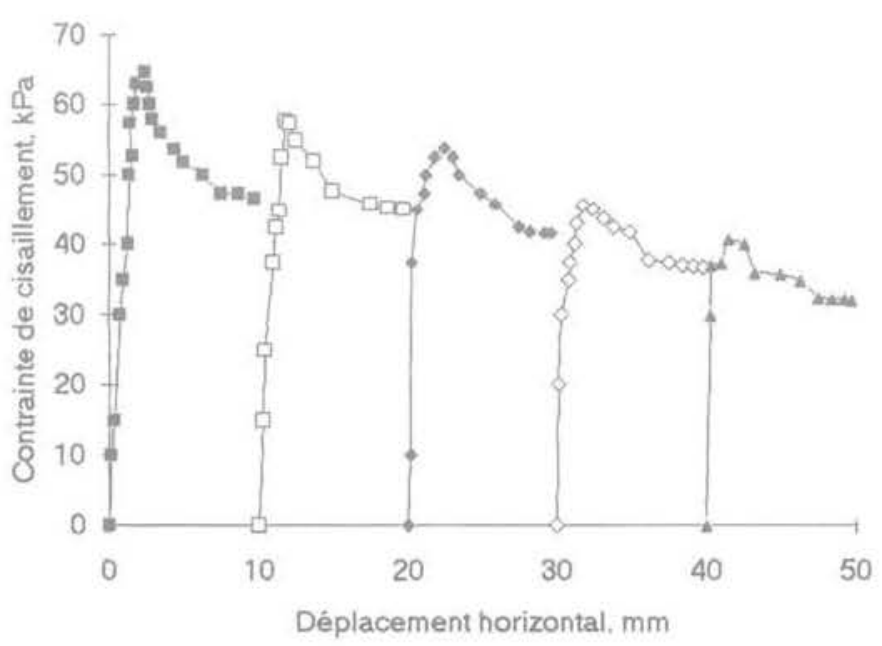

Cyles d'aller

Forward cycles

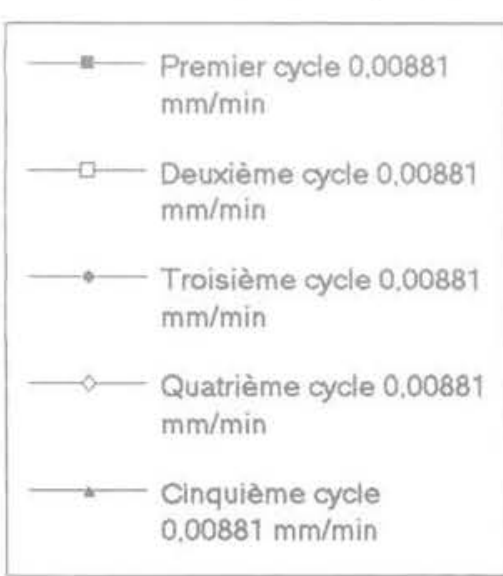

FG.1 Contrainte de cisaillement résiduelle, avec vitesse de cisaillement croissante pour l'argile de Londres. Residual shear strenght with increasing rate of shear for London clay. 
Pour les deux échantillons étudiés, la réduction de la résistance résiduelle peut être globalement expliquée par la réduction de l'effet de la perturbation de l'échantillon durant le mouvement inverse de la boîte. Il est vraisemblable que la zone de cisaillement est mieux définie en utilisant cette technique qu'avec la méthode usuellement utilisée.

Les effets de la vitesse de cisaillement ont été étudiés pour différentes valeurs de la vitesse de déplacement à chaque cycle de retour, la vitesse étant croissante au cours de chaque cycle. Cependant, au cours de chaque cycle d'aller, la vitesse gardait une valeur constante et suffisamment faible pour que les conditions résiduelles soient établies (voir Fig. 1 et 2).
En général, la résistance ne subit que de faibles variations sur les trois premiers cycles de retour pour lesquels la vitesse de cisaillement est croissante. Ces résultats sont différents de ceux correspondant aux trois premiers cycles d'aller où la résistance décroît avec chaque cycle. Ceci est cependant en accord avec les observations faites par Garga (1970). Un autre facteur, qui complique également la situation et qui est difficile à éviter, est la perte de matériau, peu considérable mais continue, à travers l'ouverture entre les deux parties de la boîte.

Ce processus est relativement indépendant du déplacement, mais si des vitesses suffisamment élevées sont utilisées, il est possible que la perte de matériau
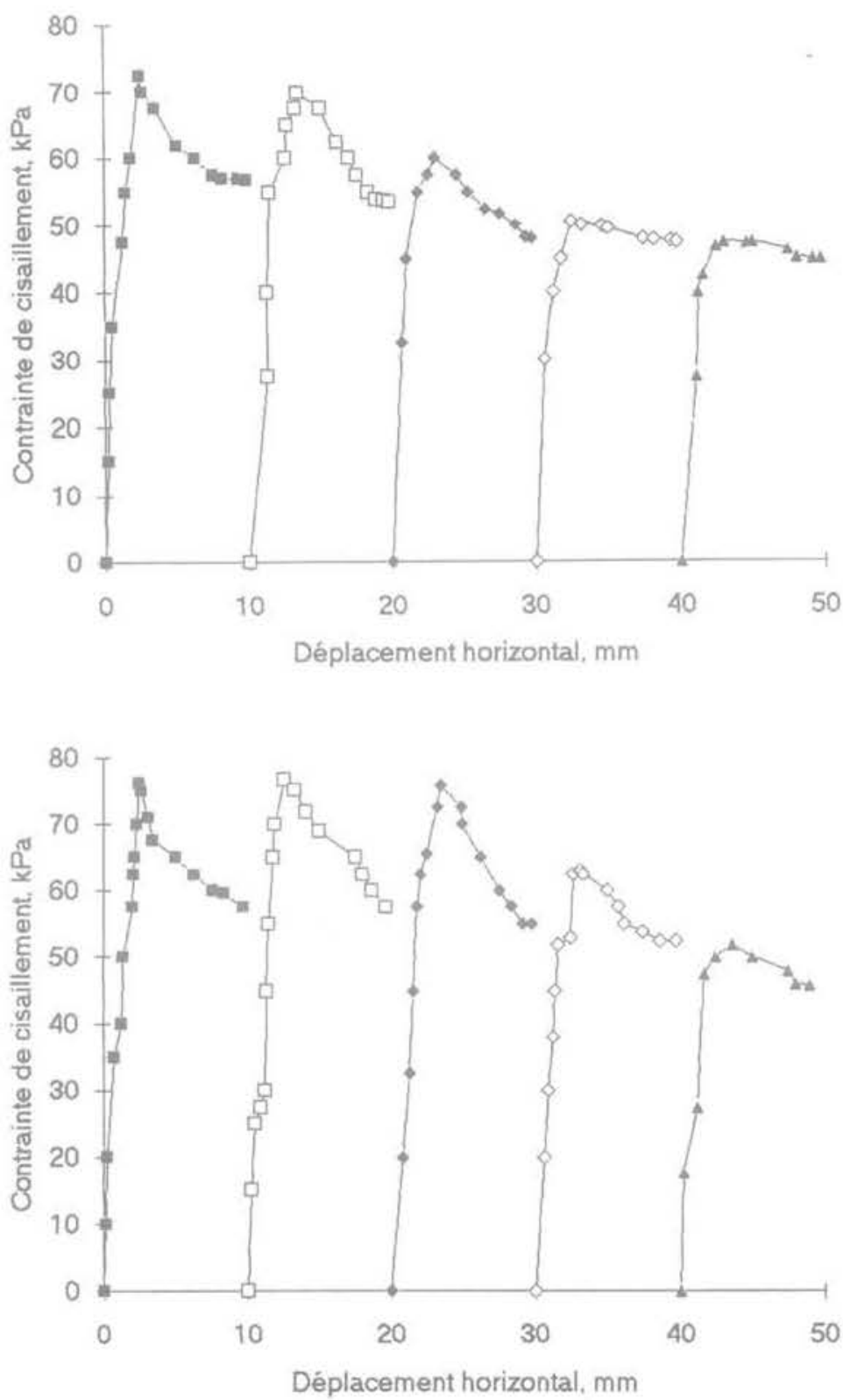

\section{Cycles d'aller}

Forward cycles

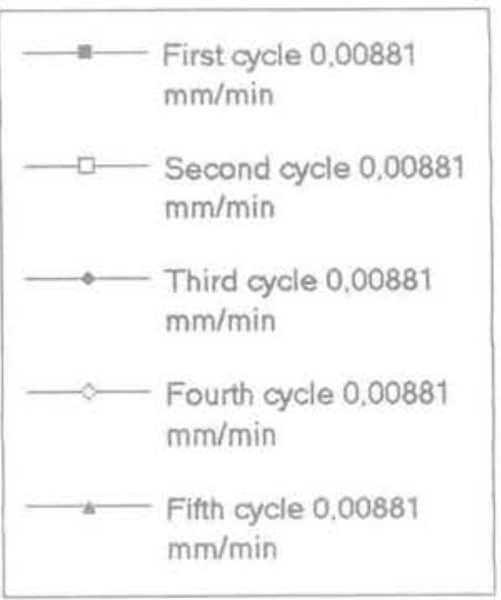

50

Cycles de retour

Backward cycles

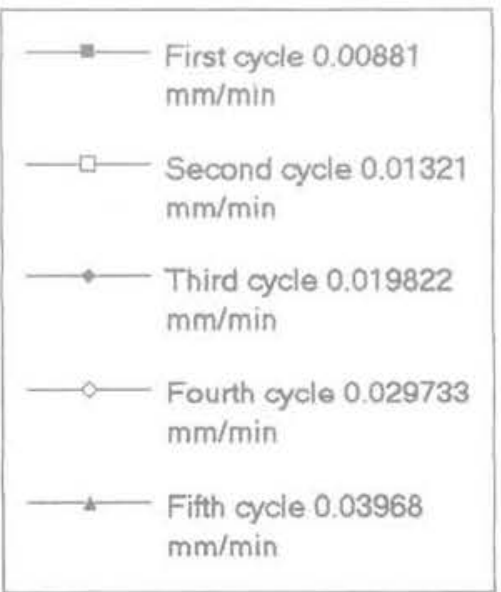

FIG. 2 Contrainte de cisaillement, avec vitesse de cisaillement croissante pour I'argile du Lias. Residual shear strenght with increasing rate of shear for Lias clay. 
peut dans une certaine mesure influencer le comportement de la pression interstitielle (Lupini, 1981). Il s'est révélé qu'après les trois premiers cycles, la chute de résistance pourrait être attribuée à un changement significatif de la structure. Les vitesses élevées produisent une dislocation dans l'orientation des particules argileuses plates, les " platelets d'argile ».

La faible variation de la résistance observée sur les trois premiers cycles de retour peut être à l'origine de l'apparition de nouvelles surfaces de glissement le long de la zone de cisaillement, car les particules argileuses plates ne sont plus en mesure de rester parfaitement parallèles comme dans le cas de faibles vitesses.

La chute de résistance après trois cycles est alors probablement due à la disparition du désordre induit dans les cycles initiaux, et l'alignement de la zone de cisaillement devient bien défini. Il y a une augmentation de la résistance au cisaillement résiduelle pour "l'argile de Londres $v$ et pour " l'argile du Lias » par rapport aux valeurs obtenues sans augmentation de la vitesse de cisaillement. La différence est de 0,85 degrés et 1,4 degrés pour « l'argile de Londres $»$ et « l'argile du Lias i) respectivement.

En conclusion, une forte vitesse de cisaillement perturbe le plan de cisaillement et cette perturbation augmente la résistance de l'échantillon. Toutefois, il y a simultanément une destruction de la cohésion et après un certain déplacement les liaisons entre particules sont rompues, si bien que la résistance diminue.

Il ressort des résultats qu'on trouve dans la littérature ainsi que des résultats expérimentaux de la présente section que les deux argiles considérées présentent un cisaillement de type glissement car elles manifestent une légère augmentation de leur résistance résiduelle avec la vitesse de cisaillement. Lupini (1981) a montré que les sols qui présentent un cisaillement résiduel de type glissement manifestent, en général, une légère augmentation de leur résistance résiduelle avec la vitesse de cisaillement, alors que les sols avec un mode de cisaillement « turbulent » ont tendance à présenter une diminution de leur résistance résiduelle avec la vitesse de cisaillement.

\section{Influence de la teneur en eau}

Une détermination précise de la teneur en eau dans la zone de cisaillement est difficile à obtenir à cause de la faible taille de cette zone. En d'autres termes, des échantillons très minces extraits du plan de cisaillement peuvent renfermer une proportion de sol non cisaillé. Pour cette raison, les résultats obtenus sur des échantillons préparés au laboratoire sont les plus sensibles.

Le taux d'humidité final de la zone de cisaillement est déterminé à l'aide de la moyenne de cing valeurs mesurées. Ces valeurs montrent que dans la zone de cisaillement, les argiles d'Algérie ont le plus haut taux d'humidité.

En dépit de certains points communs dans les caractéristiques des argiles étudiées (voir tableau V), l'effet des conditions d'environnement peut être une explication possible des différences constatées. La teneur en eau finale ne semble présenter aucune corrélation avec l'angle de frottement résiduel comme le montre la figure 3 .

\section{1}

\section{Relation entre les angles de frottement drainés, les limites d'Atterberg et la composition en argile}

L'objet de cette note est d'attirer l'attention sur une corrélation pratique qui semble exister entre l'angle de frottement résiduel, les limites d'Atterberg et la composition en argile. En général, il y a une diminution de $\varnothing_{r}^{\prime}$ lorsque la teneur en argile augmente car cette augmentation facilite la réorientation rapide des particules d'argile vers la zone de cisaillement (voir Fig. 4), Dans les figures 5 et 6 , l'angle de frottement résiduel est représenté en fonction de la limite de liquidité et de l'indice de plasticité respectivement. Comme le montre la figure 6 , il existe une bonne corrélation entre l'indice

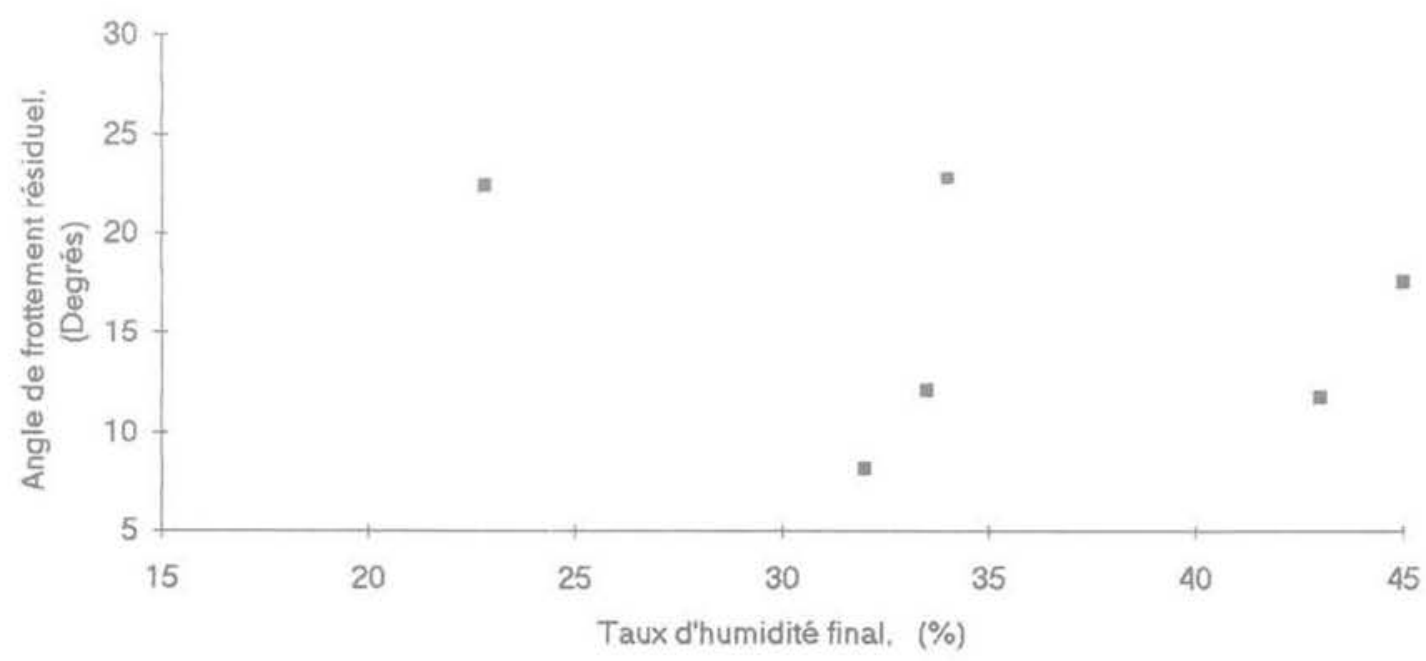

FiG.3 Angle de frottement résiduel, pas de corrélation avec le taux d'humidité final. Residual friction angle, no correlation with final moisture content. 


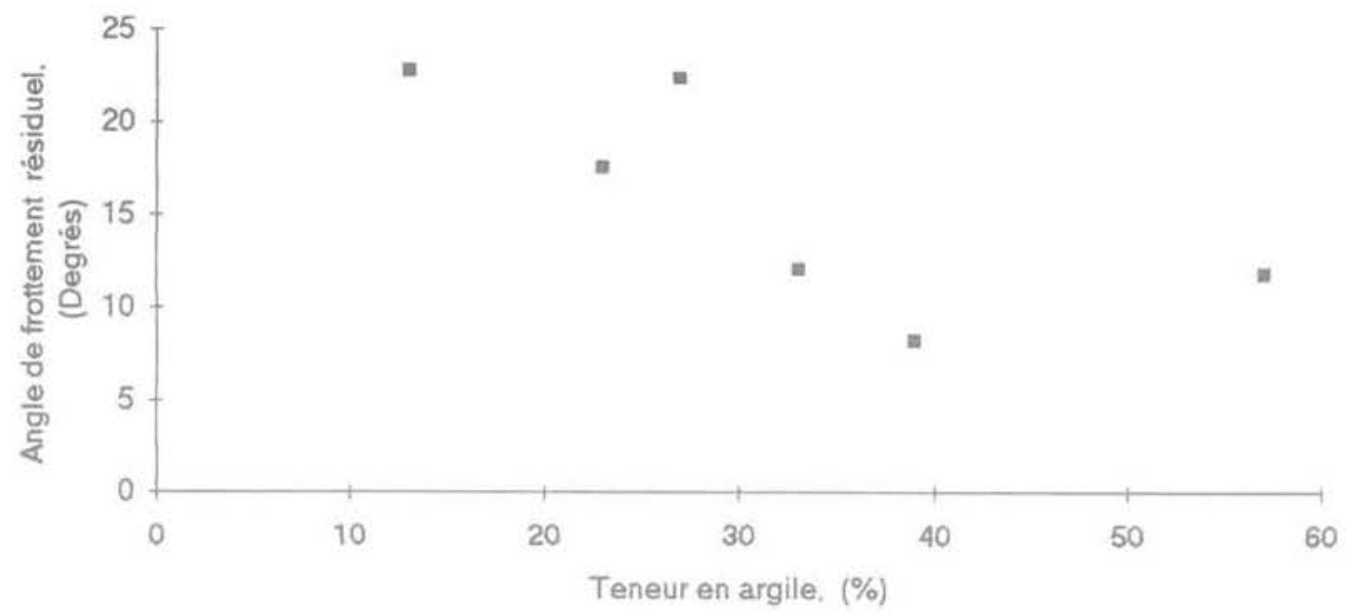

AG. 4 Angle de frottement résiduel, corrélation avec la teneur en argile. Residual friction angle, correlation with clay fraction.

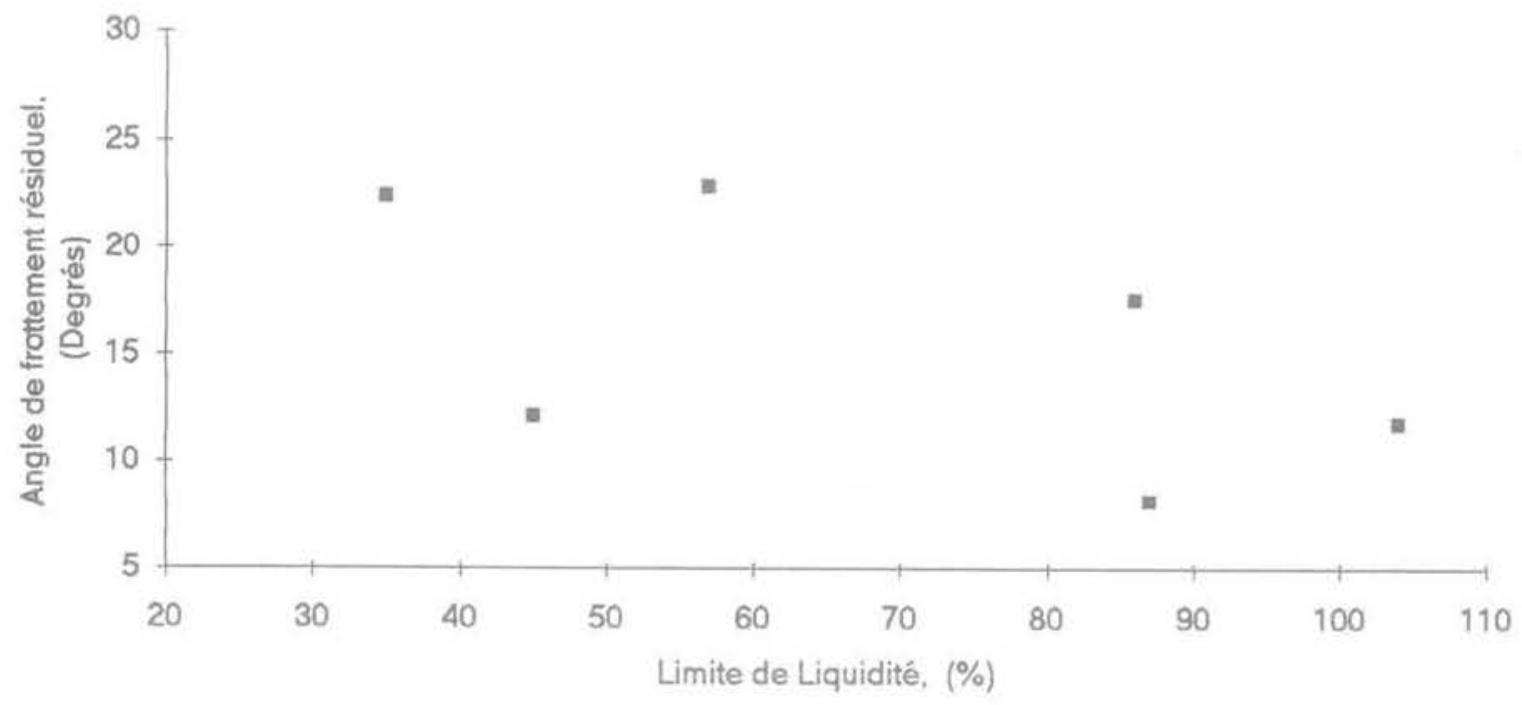

F6.5 Angle de frottement résiduel, faible corrélation avec la limite de liquidité. Residual friction angle, weak correlation with liquid limit.

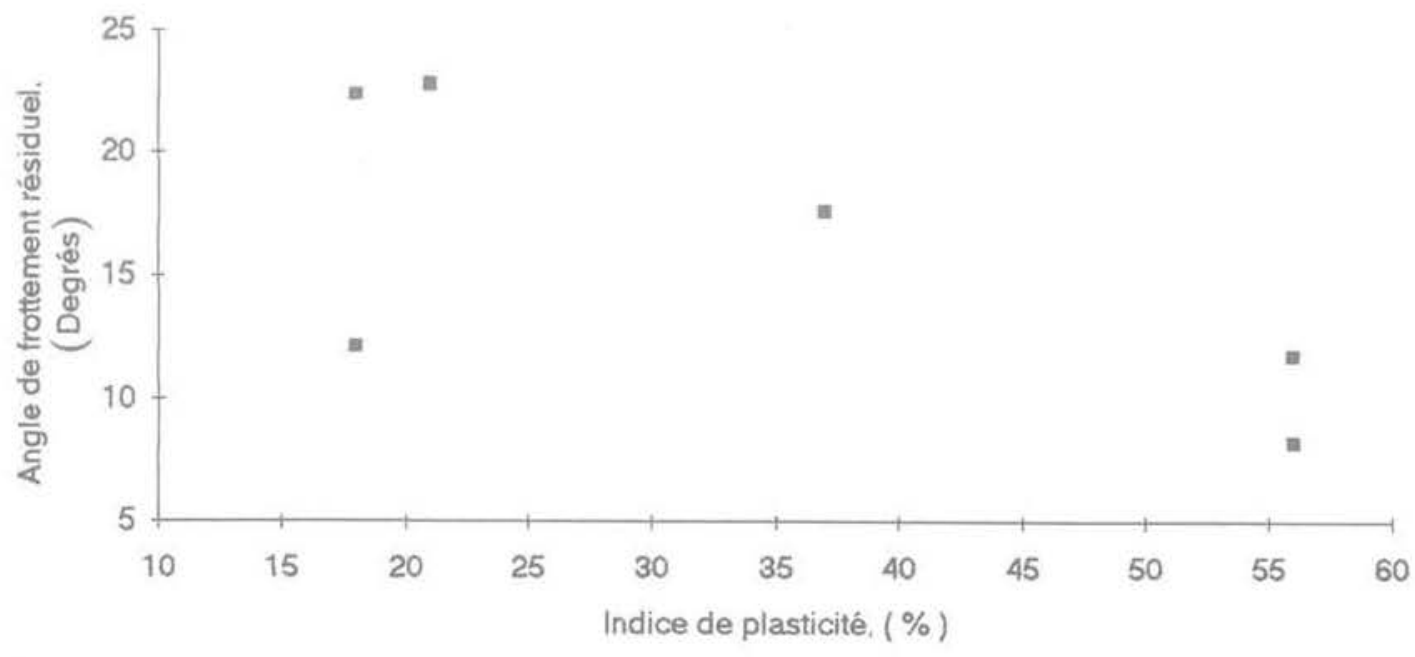

F6.6 Angle de frottement résiduel, corrélation avec l'indice de plasticité.

Residual friction angle, correlation with Plasticity Index. 
de plasticité et l'angle de frottement résiduel (observer que la variation de l'angle de frottement est sensiblement linéaire sauf dans le cas de «l'argile du Lias »). La corrélation entre la limite de liquidité et l'angle de frottement résiduel est par contre moins importante (Fig. 5). Il est donc clair que la corrélation entre $\varnothing^{\prime}$, et les limites d'Atterberg existe car aussi bien l'indice de plasticité que la limite de liquidité dépendent de la taille des grains et les particules argileuses plates.

\section{7}

\section{Conclusions}

Cette étude a permis de tirer les conclusions suivantes :

- la possibilité de perturbations de la surface de rupture au cours du mouvement inversé, qui est un des inconvénients de la boîte de cisaillement, a été minimisée en allégeant la charge normale pendant la phase de retour:

- la réduction des perturbations durant l'inversion de la direction du cisaillement réduit la valeur de l'angle de résistance au cisaillement résiduelle de 0,3 et
0,27 degrés pour l'argile de Londres et l'argile du Lias respectivement. Ce qui est pratiquement négligeable;

- les essais effectués avec la boîte de cisaillement sans chargement lors de l'inversion du mouvement de la boîte sont recommandés afin de réduire le plus possible la perturbation des échantillons ;

- la teneur en argile et l'indice de plasticité ont une influence significative sur l'angle de frottement résiduel et représentent des facteurs importants dans la caractérisation de la résistance résiduelle des argiles ;

- la corrélation entre le taux d'humidité et l'angle de frottement résiduel n'est pas effective.

\section{8}

\section{Remerciements}

Ce travail a été effectué au département de Génie Civil à Loughborough University of Technology, l'auteur exprime toute sa reconnaissance à l'administration du département pour les facilités en équipements et la disponibilité des techniciens, ce qui a considérablement aidé à la réalisation de ce projet.

\section{Bibliographie}

Blondeau F, et Josseaume H. - Mesure de la résistance au cisaillement résiduelle en laboratoire. Bulletin de liaison des laboratoires des ponts et chaussées, stabilité des talus, versants naturels, Numéro spécial II, 1976, p. 90-106.

Hawkins A.B. et Privett K.D. - Discussion on the residual shear strength of cohesive soils. Ground Engineering, $18, n^{\circ} 8$, 1985, p. $22-29$.

Hermann H.G. et Wolfskill L.A. - Residus shear strength of weak shales. Technical report, $n^{\circ} 3-699$, Engineering properties of nuclear craters, report 5 . Cam- bridge, Massachussets Institute of technology, 1966, $230 \mathrm{p}$.

Kenney T.C. - The influence of mineral composition on the residual strength of natural soils. Proceedings of geotechnical conference, Oslo 1. Norwegian Geotechnical Institute, 1967, p. 123-129.

Lupini J.F., Skinner A.E. et Vaughan P.R. The drained residual strength of cohesive soils, Geotechnique $19, \mathrm{n}^{\circ} 3,1981$, D. $321-334$

Rogers C.D.F, Boyce J,R. et Rouaiguia A. A fully automated shearbox for residual strength measurement. Proceedings second international conference on foundations and tunnels, University of Edinburgh, United Kingdom, volume 2. 1989, p. 245-251.

Rouaiguia A. - Strength of soil-structure interfaces. Ph. D. thesis, Loughborough University of Technology, United Kingdom, 1990, 278 p.

Skempton A.W. - Long-term stability of clay slopes. Fourth Rankine lecture, Geotechnique, vol. XIV, $n^{\circ} 2,1964$, p. 77 101

Voight B. - Correlation between Atterberg plasticity limits and residual shear strength of natural soils. Geotechnique 27. $n^{\circ} 2,1973$, p. $125-136$. 